\title{
A 5-Year Review of Gynaecological Oncology Patients Managed by a Fast Track Surgery Program
}

\author{
Jonathan Carter ${ }^{1,2,3}$, Shannon Philp ${ }^{1,2,3}$, Rachel O’Connell ${ }^{2,3,4}$ \\ ${ }^{1}$ Sydney Gynaecological Oncology Group, Sydney, Australia; ${ }^{2}$ Sydney Cancer Centre, Royal Prince Alfred Hospital, Sydney, Aus- \\ tralia; ${ }^{3}$ The University of Sydney, Sydney, Australia; ${ }^{4}$ NHMRC Clinical Trials Centre, Sydney, Australia. \\ Email: j.carter@sydney.edu.au \\ Received December $6^{\text {th }}, 2013$; revised January $3^{\text {rd }}, 2014$; accepted January $15^{\text {th }}, 2014$ \\ Copyright (c) 2014 Jonathan Carter et al. This is an open access article distributed under the Creative Commons Attribution License, \\ which permits unrestricted use, distribution, and reproduction in any medium, provided the original work is properly cited. In accor- \\ dance of the Creative Commons Attribution License all Copyrights ¿ 2014 are reserved for SCIRP and the owner of the intellectual \\ property Jonathan Carter et al. All Copyright (C) 2014 are guarded by law and by SCIRP as a guardian.
}

\section{ABSTRACT}

Introduction: The aim of this study is to provide a comprehensive 5-year audit of patients undergoing laparotomy for suspected or confirmed gynaecological malignancy to document the frequency and incidence of adverse events and to investigate factors associated with shorter length of stay and readmission to hospital. Methods: A 5-year surgical audit of the period commencing 2008 and concluding 2012. All patients undergoing laparotomy were included in the audit without exclusions. Approval was granted by the local Ethics Review Committee. Results: Four hundred and twenty-seven patients underwent laparotomy for suspected or confirmed gynaecological malignancy and were managed by Fast Track Surgery (FTS) principles. Average age was 54.8 years and average weight and BMI were $73.4 \mathrm{~kg}$ and 28.1 respectively. Ultimately 254 (59\%) patients had confirmed malignancy. Average surgery duration was 2.36 hours and average estimated blood loss (EBL) at surgery was $262 \mathrm{~mL}$. Median and mean LOS was 3.0 and 3.5 days respectively with $125(29 \%)$ patients discharged on day 2. Overall transfusion rate was $5 \%$. Other adverse events in decreasing frequency were hospital readmission (3.7\%), significant wound infection (3\%) and unplanned High Dependency Unit (HDU) admission (1.4\%). All other adverse events were uncommon with rates $<0.5 \%$. Factors associated with a short LOS included year of surgery, age, performance status, malignant vs benign pathology, the use of COX-2 inhibitors, operation time, incision type, transfusion, and radical hysterectomy, at least 1 complication, if patients tolerated early oral feeding (EOF). In multivariable analysis, year, age, performance status, the use of COX-2 inhibitors, operation time and incision type were significant. Factors associated with readmission included the use of COX-2 inhibitors, operation time, performance of a lymph node dissection, return to operating theatre, operation category at least 1 complication, and in multivariable analysis lymph node dissection and the occurrence of at least 1 complication were significant. Conclusions: This 5-year audit is important in establishing a contemporary incidence and the prevalence rate of serious adverse events for patients with suspected or confirmed gynaecological cancer undergoing laparotomy and managed by FTS principles. The community can be reassured that the incidence of serious adverse events is low when managed by FTS principles.

\section{KEYWORDS}

Fast Track Surgery; Enhanced Recovery; Optimized Recovery

\section{Introduction}

Fast Track Surgery (FTS) programs are also known as Enhanced Recovery after Surgery (ERAS) or Optimized Recovery after Surgery (ORAS) programs. They were first described by Kehlet in Denmark [1] and the general principles have been adopted by most surgical specialties worldwide $[2,3]$. The basic tenet of such programs is to enhance recovery after surgery, allowing earlier discharge with improved patient outcomes and low readmission rates. Such programs derive their success from being multidisciplinary with all members of the team having an important function. Team members include 
surgeons, nurses, anaesthetist, pain specialists, ward nursing staff, social worker, occupational and physical therapy staff $[4,5]$. There is no long term published data on the safety and outcomes of such programs in gynaecological oncology.

The aim of this manuscript is to provide a comprehensive 5-year audit of patients undergoing laparotomy for suspected or confirmed gynaecological malignancy managed by a FTS or ORAS program, to document the frequency and incidence of adverse events, length of stay (LOS) and readmission rates with factors contributing to these.

\section{Materials and Methods}

An audit of the author's surgical practice (laparotomy) was performed for the 5-year period commencing 2008 and concluding 2012. All patients undergoing laparotomy were included in the audit without exclusions. Approval was granted by our local Ethics Review Committee. The performance of the FTS or ORAS program has previously been reported [6,7].

Patient characteristic data collected includes age, comorbidities, previous intra-abdominal surgery, weight, height, body mass index (BMI), medical insurance status, ASA score and performance status. Hospitalization details included the procedure performed, type of incision (transverse or midline), operating time, complexity of surgery (simple vs. complex), operation category (elective vs. emergency), wound infection risk (clean vs. clean contaminated vs. contaminated), intraoperative estimated blood loss (EBL), whether a transfusion was required, the preoperative haemoglobin $(\mathrm{Hb})$, post operative $\mathrm{Hb}$ and the $\mathrm{Hb}$ change, whether patients tolerated early oral feeding (EOF) and if the patient received cyclooxygenase inhibitors (COX Inhibitors). All inpatient complications were collected, including modified Royal Australian and New Zealand College of Obstetricians and Gynecologists (RANZCOG) Quality Indicators. Date of surgery and date of discharge were used to calculate LOS. Post hospitalization admissions and complications were also recorded. Not all data fields were collected from initiation of the audit. In year 3 "Suitability for D/C on day 2" was included. From year 4 the following data fields were added to the audit: "ASA score", "comorbidities", "previous intra-abdominal surgery", "operation category", "wound infection risk".

Simple surgery was defined as simple type 1 hysterectomy or adnexal surgery where formal retroperitoneal dissection or ureteric dissection was not performed. All surgeries where at least a formal pelvic sidewall dissection was undertaken, including bowel, bladder, nodal dissection and omentectomy were classified as "complex". Transverse incisions were classified according to the incision in the skin, irrespective of whether it was of
Maylard or Pfannenstiel type.

Patients were classified on final pathological determination as either "benign" or "malignant". Patients with proliferating or borderline ovarian tumours were classified as "benign" as were patients with complex atypical endometrial hyperplasia and patients with cervical dysplasia needing definitive treatment and patients undergoing definitive hysterectomy for persistent gestational trophoblastic disease.

Statistical analysis included descriptive statistics, bivariate analysis included t-test and ANOVA for nominal variables and chi-squared test for categorical data. Logistic regression analysis was performed to determine independent predictors of LOS no greater than 2 days and hospital readmission within 30 days of surgery.

\section{Results}

Over the 5-year time period, 427 patients underwent laparotomy for high-risk gynaecologic pathology, suspected or confirmed malignancy. Average age was 54.8 years, with 155 (36\%) patients aged between 50 - 65 years, 85 (20\%) aged between 65 - 75 years and 28 (7\%) aged over 75 years. Average weight was $73.4 \mathrm{~kg}$ (Range: 38 - $192 \mathrm{~kg}$ ), average body mass index (BMI) was 28.1 (Range: 16.9 - 68.8) with 260 (61\%) patients classified as either overweight or obese. In 200873 (17\%) of laparotomies were performed, 99 (23\%) in 2009, 79 (19\%) in 2010, 96 (22\%) in 2011 and 80 (19\%) in 2012. Two hundred and eighty one (66\%) patients had zero performance status (PS) meaning they were fully active; 102 (24\%) had a PS of 1 indicating restriction in physically strenuous activity but ambulatory and able to carry out work of a light or sedentary nature, e.g., light house work, office work, and $44(10 \%)$ had a PS of 2 or 3 indicating they were ambulatory and capable of all self-care but unable to carry out any work activities (PS 2) or up and about more than $50 \%$ of waking hours or capable of only limited self-care, confined to bed or chair more than $50 \%$ of waking hours (PS 3). One hundred and eighty two (43\%) patients had previous abdominal surgery. Ovarian, corpus and cervical pathology were the most common tumour sites, accounting for 50\%, 39\% and 9\% respectively. The majority of incisions were vertical midline, occurring in 385 (90\%) and 386 (90\%) were classified as having complex surgeries performed. Ultimately 254 (59\%) patients had confirmed malignancy. Average surgery duration was 2.36 hours (Range: 0.75 - 10 hours). Average estimated blood loss (EBL) at surgery was 262 $\mathrm{mL}$ (Range $10-2500 \mathrm{~mL}$ ). Median and mean LOS was 3.0 and 3.5 days respectively with 125 (29\%) patients discharged on day 2. Overall 16 patients (3.7\%) required hospital readmission (Table 1).

During the 5-year audit period, there were $2(0.5 \%)$ bladder injuries. The first occurring in a 46-year-old 
Table 1. Detailed summary and subgroup analysis.

\begin{tabular}{|c|c|c|c|c|c|c|c|c|c|}
\hline & All & Benign & Malignant & $\begin{array}{c}\text { Ovarian } \\
\text { Cancer }\end{array}$ & $\begin{array}{l}\text { Uterine } \\
\text { Cancer }\end{array}$ & $\begin{array}{l}\text { Cervical } \\
\text { Cancer }\end{array}$ & Obese & $\begin{array}{c}\text { Elderly } \\
(\geq 75)\end{array}$ & $\begin{array}{c}\text { D/C } \\
\text { Day } 2\end{array}$ \\
\hline $\mathbf{N}$ & 427 & 173 & 254 & 98 & 126 & 24 & 137 & 28 & 125 \\
\hline Age (years) & 54.8 & 50 & 58.1 & 57.2 & 63.4 & 43.6 & 57.3 & 79.8 & 50.2 \\
\hline Complex & $90 \%$ & $79 \%$ & $98 \%$ & $98 \%$ & $98 \%$ & $96 \%$ & $93 \%$ & $96 \%$ & $86 \%$ \\
\hline Non Zero Performance Status & $34.2 \%$ & $18.5 \%$ & $44.5 \%$ & $43.9 \%$ & $47.6 \%$ & $4.2 \%$ & $55 \%$ & $93 \%$ & $19 \%$ \\
\hline ASA $>1$ & $62.5 \%$ & $43 \%$ & $75.5 \%$ & $86 \%$ & $78 \%$ & $33.3 \%$ & $81 \%$ & $100 \%$ & 49.3 \\
\hline Vertical Incision & $90.2 \%$ & $81.5 \%$ & $96.1 \%$ & $98 \%$ & $99 \%$ & $70.8 \%$ & $99 \%$ & $100 \%$ & $78 \%$ \\
\hline Weight (kg) & 73.4 & 73.5 & 73.3 & 67.4 & 80.2 & 63.5 & 95.4 & 71.7 & 71.5 \\
\hline BMI & 28.1 & 28 & 28.3 & 25.9 & 31 & 24.5 & 36.6 & 29.2 & 27 \\
\hline Obese & $32 \%$ & $32 \%$ & $32 \%$ & $18 \%$ & $46 \%$ & $17 \%$ & $100 \%$ & $36 \%$ & $26 \%$ \\
\hline Overweight \& Obese & $60.1 \%$ & $61.9 \%$ & $60 \%$ & $47 \%$ & $77 \%$ & $37.5 \%$ & - & $82 \%$ & $54 \%$ \\
\hline EBL (mL) & 262 & 237 & 279 & 375 & 207 & 246 & 251 & 196 & 180 \\
\hline OR Time (hours) & 2.36 & 2.10 & 2.53 & 2.57 & 2.33 & 3.0 & 2.41 & 2.08 & 2.03 \\
\hline LOS Mean (days) & 3.5 & 3.1 & 3.7 & 4.1 & 3.3 & 3.3 & 3.6 & 4.4 & 2 \\
\hline LOS Median (days) & 3 & 3 & 3 & 3 & 3 & 3 & 3 & 3 & 2 \\
\hline LOS $<2 D$ & $29.3 \%$ & $43 \%$ & $20 \%$ & $16 \%$ & $21 \%$ & $25 \%$ & $24 \%$ & $3.6 \%$ & $100 \%$ \\
\hline Readmission Rate & $3.7 \%$ & $2.3 \%$ & $4.7 \%$ & $5.1 \%$ & $5.6 \%$ & 0 & $5.1 \%$ & $7 \%$ & $1.6 \%$ \\
\hline Return OR & $0.5 \%$ & 0 & $0.8 \%$ & 0 & $1.6 \%$ & 0 & $0.7 \%$ & $0 \%$ & $0.8 \%$ \\
\hline Ureteric Injury & 0 & 0 & 0 & 0 & 0 & 0 & 0 & $0 \%$ & 0 \\
\hline Bladder Injury & $0.47 \%$ & $0.6 \%$ & $0.4 \%$ & 0 & $0.8 \%$ & 0 & $1.5 \%$ & $0 \%$ & $0.8 \%$ \\
\hline Bowel Injury & 0 & 0 & 0 & 0 & 0 & 0 & 0 & $0 \%$ & 0 \\
\hline Wound Infection & $3 \%$ & $2.3 \%$ & $3.5 \%$ & $1 \%$ & $4.8 \%$ & 4.2 & $6.6 \%$ & $3.6 \%$ & $2.4 \%$ \\
\hline Unplanned ICU Admit & $1.4 \%$ & $0.6 \%$ & $2 \%$ & $1 \%$ & $2.4 \%$ & $4.2 \%$ & $2.2 \%$ & $3.6 \%$ & $0.8 \%$ \\
\hline VTE & $0.9 \%$ & $0.6 \%$ & $1.2 \%$ & $1 \%$ & $1.6 \%$ & 0 & $2.2 \%$ & $3.6 \%$ & $0.8 \%$ \\
\hline Transfusion & $5.2 \%$ & $3 \%$ & $6 \%$ & $13.3 \%$ & $1.6 \%$ & 0 & $3.7 \%$ & $3.6 \%$ & $1.6 \%$ \\
\hline Preop & $2.1 \%$ & $1 \%$ & $2 \%$ & $5 \%$ & $1.6 \%$ & 0 & $2.9 \%$ & 0 & $0.8 \%$ \\
\hline Intra/Post & $3.3 \%$ & $2 \%$ & $4 \%$ & $9 \%$ & 0 & 0 & $1.5 \%$ & $3.6 \%$ & $0.8 \%$ \\
\hline
\end{tabular}

obese woman (BMI > 32) undergoing a total abdominal hysterectomy bilateral salpingoophorectomy (THBSO) for an enlarged uterus. There was poor surgical access and she had had 2 prior cesarean sections. The injury was immediately identified and repaired without complication. The second bladder injury occurred in a 61-year-old obese woman (BMI > 30) who underwent a pelvic exenteration. She was a Jehovah's Witness, and had previously undergone an abdomino-perineal resection and pelvic irradiation for colon cancer, re-presenting with a serous corpus cancer with her uterus fixed to the sacrum. The bladder was morbidly fixed to the uterus and was inadvertently entered during dissection. The injury was immediately identified and repaired in 2 layers. Her perioperative course was complicated by bleeding from ureteric stents and febrile morbidity, resulting in her return to the operating theatre for a check cystoscopy. She was subsequently readmitted with impaired renal function, settling without significant intervention.

Two patients had unplanned return to the operating room (OR). The first outlined above. The other patient who was returned to the OR was 46-year-old overweight woman (BMI > 29) with a stage III uterine cancer who underwent a THBSO/lymph node dissection (LND), whose surgery was prolonged, lasting for $3 \mathrm{hrs} 15 \mathrm{~min}$. She was readmitted after discharge on day 2 with a wound infection, requiring return to the OR for debridement.

Six (1.4\%) patients had unplanned admissions to High Dependency Unit/Intensive Care Unit (HDU/ICU). Two patients had unexplained intraoperative bradycardia and were observed overnight in HDU. The patient described above for monitoring for bleeding and renal impairment. 
Two patients were admitted for observation with impaired respiratory effort post extubation and a 72-yearold woman for observation after primary debulking surgery for ovarian cancer requiring a posterior exenteration, surgery lasting $>5$ hours and blood loss of $2500 \mathrm{~mL}$.

Thirteen (3\%) patients developed clinical wound infections classified as greater than grade 2 requiring some sort of intervention. Blood transfusion rates are considered "a general measure of surgical management" [8]. Overall 22 (5\%) patients received blood transfusions. Table 2 expands upon this global figure with only 14 (3\%) receiving intraoperative or postoperative transfusions and only $4(0.9 \%)$ receiving greater than 2 units.

Sixteen $(3.7 \%)$ of patients were readmitted, 4 with wound infection, 3 with constipation and one each of the following: resuturing of vaginal vault, elevated creatinine, dehydration, haematoma, gastroenteritis, trial of void, gall stones, pancreatitis and ileus.

Four $(0.9 \%)$ patients had confirmed venous thrombo embolism (VTE). It is noteworthy that 3 of VTEs were diagnosed on preoperative imaging and the 4th patient underwent a posterior exenteration for ovarian cancer after neoadjuvant chemotherapy and developed a VTE after discharge.

Overall and subset analysis of outcomes is shown in Table 1 including incidence of adverse events and Quality Indicators, whist Table 2 summarizes and clarifies transfusion rates.

Obese patients $(\mathrm{BMI}>30)(\mathrm{N}=137)$ when compared to the patients with normal BMI (BMI < 25) $(\mathrm{N}=167)$ and overweight patients (BMI 25 - 30) $(\mathrm{N}=123)$ were significantly more likely to be older $(\mathrm{P}<0.0002)$, have corpus cancers $(\mathrm{P}<0.04)$ have non-zero performance status $(\mathrm{P}<0.0001)$, ASA $>1(\mathrm{P}<0.002)$, have VMI's performed $(\mathrm{P}<0.0001)$. They also had higher wound infection rates $(\mathrm{P}<0.02)$ but due to low numbers the statistical significance of this is questioned. Obese patients did not have a significantly different rate of complex surgeries performed, blood transfusions, blood loss at surgery, LOS, readmission rate, VTE, return to theatre or bladder injury (Table 1 ).

Elderly patients (Age $>75$ years) were significantly more likely to have VMI $(\mathrm{P}<0.002)$, have ASA $>1$ ( $\mathrm{P}<$

Table 2. Blood transfusion rates and description.

\begin{tabular}{cc}
\hline \multicolumn{2}{c}{ Blood Transfusion } \\
\hline Indication & Incidence \\
Overall & $22^{*}(5 \%)$ \\
Preoperative & $9(2 \%)$ \\
Intra \& Postoperative & $14(3 \%)$ \\
Transfusion > 2 Units & $4(0.9 \%)$ \\
\hline
\end{tabular}

* One patient received both pre and intraoperative transfusion.
0.0001), have a non-zero performance status ( $\mathrm{P}<0.0001)$ undergo complex surgeries $(\mathrm{P}<0.02)$, to be obese or overweight $(\mathrm{P}<0.04)$, have a longer length of stay $(\mathrm{P}<$ 0.02). Elderly patients had operation durations not significantly different to those less than 50 years, aged between 50 - 65 or 65 - 75 years, were not more likely to be transfused nor have increased blood loss at surgery, wound infection, ureteric or bladder injury rate, nor be readmitted, return to the operating room or have an unplanned ICU admission (Table 1).

Factors associated with a significant short LOS on univariate analysis included year of surgery, age, performance status, malignant verses benign pathology, use of COX-2 inhibitors, operation time, incision type, transfusion, radical hysterectomy, at least 1 complication and the patient's tolerance of EOF. On multivariable analysis year, age, performance status, use of COX-2 inhibitors, operation time and incision type were significant.

Factors associated with hospital readmission included use of COX-2 inhibitors, operation time, performance of a lymph node dissection, return to operating theatre, operation category and at least 1 complication. On multivariate analysis, lymph node dissection and the occurrence of at least 1 complication were significant $(\mathrm{P}<0.05)$.

\section{Discussion}

Surgeons are constantly striving to improve outcomes for their patients. Whilst minimally invasive surgery is becoming increasingly popular, laparotomy currently remains the standard of care for management of the majority of patients with gynaecological cancers. However the improved short-term outcomes achieved with minimally invasive surgery has almost forced surgeons undertaking laparotomy to evaluate and adopt strategies to favourably complete with those enhanced short-term outcomes. One such strategy is "Fast Track Surgery". The author initiated such a program at a major University Teaching Hospital and commensurate with the principles of clinical governance, has regularly undertaken clinical audit of the program to attest to its ongoing safety and efficacy [6,7,9-13]. The data presented in this clinical audit represents actual and all surgical performance outcomes, without prejudice, exclusions or exceptions and is the largest reported series of patients with suspected or confirmed gynaecological cancer managed by FTS [14].

This 5-year clinical audit provides overwhelming support for the principles of FTS and the outcomes achieved, and attests to its overall safety and supports the view that FTS principles should become standard of care for all surgery patients.

Not only are overall LOS and readmission rates low, but complications and serious adverse events (SAE) are remarkably low when compared to contemporary reports [15]. The ureteric and bladder injury rates of $0 \%$ and $0.5 \%$ 
is lower than reported in similar studies [16] and significantly lower than accepted norms of $1.7 \%$ and $3.6 \%$ respectively $[17,18]$. The incidence of postoperative wound related issues is estimated to vary up to $10 \%$, whereas in the current audit it is $3 \%$ comparing favourably with other reported series $[19,20]$.

The reported blood transfusion rate of 5\% seems high but a large proportion of these patients underwent preoperative transfusion for pre-existing anaemia related either to blood loss or marrow suppression as a consequence of neoadjuvant chemotherapy. The 3\% intra and post operative transfusion rate compares favourably with reported rates of 7.1 per 100 patients reported by the Australian Council of Health Care Standards (ACHS) [21] and is also lower than the $6 \%$ and $9 \%$ rates for laparoscopy and laparotomy patients in the GOG study [15].

Most interesting is the very low rate of VTE of $0.9 \%$, which is lower than currently reported rates of $2 \%-4 \%$ [20-22]. The process of early ambulation and our standardized approach to VTE prophylaxis has likely contributed to this unexpected low rate. The unplanned return to the operating room of $3.7 \%$ was also consistent with previous reports $[15,20]$.

There were no deaths in the 30-day period after surgery and when compared to the LAP2 study which reported 18 deaths in the 30-day postoperative period as a consequence of thromboembolic events, reoperation, hemorrhage, progressive disease, chemotherapy and infection, this study adds further supportive evidence of the safety of the care provided and the FTS program [15].

The median and mean LOS of 3 and 3.5 days respectively in this current audit after major abdominal surgery is remarkable. Indeed these figures compare most favourably with the reported rates of the GOG. Their median length of stay for laparotomy patients was 4 days and the median length of stay for the intent-to-treat laparoscopy arm patients was 3 days. In that study, LOS greater than 2 days occurred in 52\% of laparoscopy and $94 \%$ of laparotomy patients. In this audit, LOS of greater than 2 days after laparotomy occurred in 71\% [15].

The readmission rate of $3.7 \%$ also compares favourably with accepted norms. In the GOG LAP2 study, readmission rates were $7 \%$ for laparotomy and $6 \%$ for laparoscopy [15].

Despite the author undertaking a rigorous follow-up schedule [7], critics could argue that it is possible that the incidence complications and adverse events is underreported, particularly grade 1 and 2 adverse events. The author acknowledges that this is possible, however it is unlikely that instances of major or grade 3 or 4 adverse events have been missed or underreported.

Obesity is an increasingly common problem facing surgeons and such patients have their own inherent risk of adverse events. It would appear however that apart from an increased wound infection rate, their outcome is not too dissimilar to patients with a normal BMI.

Elderly patients also have a higher rate of associated comorbidities and poorer performance status compared to younger patients and, as a consequence, are more likely to have a longer LOS.

Based upon the data presented, FTS principles should be extended to, and adopted by all general gynaecologists. Indeed, with a lower rate of complex surgeries performed by general colleagues, one could anticipate even greater advances than published here in a predominantly high risk group.

\section{Conclusion}

In conclusion whilst it is unreasonable to expect that complications will not occur after surgery, it is reasonable to expect that procedural specialists should minimize the occurrence of such events. The first step in the process is to undertake regular clinical audits, compare their outcomes with accepted current standards, identify "potential" shortcomings, take appropriate action to minimize harm and improve patient outcomes. This 5-year audit is important in establishing a contemporary incidence and the prevalence rate of serious adverse events after laparotomy managed by FTS principles for patients with suspected or confirmed gynaecological cancer. The community can be reassured that the incidence of serious adverse events is low in current surgical practice.

\section{REFERENCES}

[1] H. Kehlet, "Fast-Track Colorectal Surgery," The Lancet, Vol. 371, No. 9615, 2008, pp. 791-793. http://dx.doi.org/10.1016/S0140-6736(08)60357-8

[2] H. Kehlet, "Principles of Fast Track Surgery. Multimodal Perioperative Therapy Programme,” Der Chirurg, Vol. 80, No. 8, 2009, pp. 687-689. http://dx.doi.org/10.1007/s00104-009-1675-2

[3] H. Kehlet and D. W. Wilmore, "Multimodal Strategies to Improve Surgical Outcome," The American Journal of Surgery, Vol. 183, No. 6, 2002, pp. 630-641. http://dx.doi.org/10.1016/S0002-9610(02)00866-8

[4] F. Carli, P. Charlebois, G. Baldini, O. Cachero and B. Stein, "An Integrated Multidisciplinary Approach to Implementation of a Fast-Track Program for Laparoscopic Colorectal Surgery," Canadian Journal of Anaesthesia, Vol. 56, No. 11, 2009, pp. 837-842.

http://dx.doi.org/10.1007/s12630-009-9159-x

[5] K. Varadhan, D. N. Lobo and O. Ljungqvist, "Enhanced Recovery after Surgery: The Future of Improving Surgical Care," Critical Care Clinics, Vol. 26, No. 3, 2010, pp. 527-547. http://dx.doi.org/10.1016/j.ccc.2010.04.003

[6] J. Carter, "Fast-Track Surgery in Gynaecology and Gynaecologic Oncology: A Review of a Rolling Clinical Audit," ISRN Surgery, Vol. 2012, 2012, Article ID: 368014. http://dx.doi.org/10.5402/2012/368014 
[7] J. Carter, R. Szabo, W. Sim, et al., "Fast Track Surgery in Gynaecological Oncology: A Clinical Audit,” Australian and New Zealand Journal of Obstetrics and Gynaecology, Vol. 50, No. 2, 2010, pp. 159-163.

http://dx.doi.org/10.1111/j.1479-828X.2009.01134.x

[8] “Clinical Indicator User Manual 2012: Gynaecology Version 6,” The Australian Council on Healthcare Standards, Ultimo, 2012, pp. 1-27.

[9] J. Carter and S. Philp, "Program Development and Extended Experience with a Fast Track Surgery Program,” Australian Society of Gynaecological Oncologists Annual Scientifc Meeting, Millbrook Resort, Queenstown, 2011.

[10] J. Carter and S. Philp, "Assessing Outcomes after Fast Track Surgical Management of Corpus Cancer,” Open Journal of Obstetrics and Gynecology, Vol. 1, No. 3, 2011, pp. 139-143. http://dx.doi.org/10.4236/ojog.2011.13026

[11] J. Carter, S. Philp and V. Arora, "Fast Track Gynaecologic Surgery in the Overweight and Obese Patient," International Journal of Clinical Medicine, Vol. 1, No. 2, 2010, pp. 64-69. http://dx.doi.org/10.4236/ijcm.2010.12011

[12] J. Carter, S. Philp and V. Arora, "Early Discharge after Major Gynaecological Surgery: Advantages of Fast Track Surgery,” Open Journal of Obstetrics and Gynaecology, Vol. 1, No. 1, 2011, pp. 1-5. http://dx.doi.org/10.4236/ojog.2011.11001

[13] J. Carter, S. Philp, V. Arora, et al., "Discharge on Postop Day 2 after Major Gynaecological Surgery. Is it Possible?’ Oral Presentation 25th Annual Scientific Meeting Australian Society of Gynaecological Oncologists, Bunker Bay, 23-28 March 2010.

[14] RACS, "A Guide by the Royal Australian College of Surgeons. Surgical Audit and Peer Review,” Royal Australian College of Surgeons, Melbourne, 2008, p. 42.

[15] J. L. Walker, M. R. Piedmonte, N. M. Spirtos, et al., "Laparoscopy Compared With Laparotomy for Comprehensive Surgical Staging of Uterine Cancer: Gynecologic
Oncology Group Study LAP2,” Journal of Clinical Oncology, Vol. 27, No. 32, 2009, pp. 5331-5336. http://dx.doi.org/10.1200/JCO.2009.22.3248

[16] S. Kondalsamy-Chennakesavan, C. Bouman, S. De Jong, et al., "Clinical Audit in Gynecological Cancer Surgery: Development of a Risk Scoring System to Predict Adverse Events,” Gynecologic Oncology, Vol. 115, No. 3, 2009, pp. 329-333. http://dx.doi.org/10.1016/j.ygyno.2009.08.004

[17] S. Bai, E. Huh, D. J. Jung, et al., "Urinary Tract Injuries during Pelvic Surgery: Incidence Rates and Predisposing Factors," International Urogynecology Journal, Vol. 17, No. 4, 2006, pp. 360-364.

http://dx.doi.org/10.1007/s00192-005-0015-4

[18] B. Vakili, “The Incidence of Urinary Tract Injury during Hysterectomy: A Prospective Analysis Based on Universal Cystoscopy," American Journal of Obstetrics \& Gynecology, Vol. 192, No. 5, 2005, pp. 1599-1604. http://dx.doi.org/10.1016/j.ajog.2004.11.016

[19] M. Wechter, M. Pearlman and K. Hartmann, "Reclosure of the Disrupted Laparotomy Wound: A Systematic Review,” Obstetrics \& Gynecology, Vol. 106, No. 2, 2005, pp. 376-383. http://dx.doi.org/10.1097/01.AOG.0000171114.75338.06

[20] E. A. Erekson, S. O. Yip, M. M. Ciarleglio and T. R. Fried, "Postoperative Complications after Gynecologic Surgery,” Obstetrics \& Gynecology, Vol. 118, No. 4, 2011, pp. 785-793. http://dx.doi.org/10.1097/AOG.0b013e31822dac5d

[21] The Australian Council on Healthcare Standards (ACHS), “Australian Clinical Indicator Report 2004-2011,” 13th Edition, The Australian Council on Healthcare Standards, Ultimo, 2012.

[22] A. Peedicayil, A. Weaver, X. Li, E. Carey, W. Cliby and A. Mariani, "Incidence and Timing of Venous Thromboembolism after Surgery for Gynecological Cancer," Gynecologic Oncology, Vol. 121, No. 1, 2011, pp. 64-69. http://dx.doi.org/10.1016/j.ygyno.2010.11.038 